Congreso Internacional de Ciencias Exactas y Naturales. Universidad Nacional de Costa Rica, San José, 2019.

\title{
Uso de GeoGebra para la construcción de diagramas de cuerpo libre y editor de imágenes en la enseñanza de la Física.
}

\section{Marco Vinicio López-Gamboa y Martín Esteban Corrales Quesada.}

Cita:

Marco Vinicio López-Gamboa y Martín Esteban Corrales Quesada (2019). Uso de GeoGebra para la construcción de diagramas de cuerpo libre y editor de imágenes en la enseñanza de la Física. Congreso Internacional de Ciencias Exactas y Naturales. Universidad Nacional de Costa Rica, San José.

Dirección estable: https://www.aacademica.org/marco.lopez/19 ARK: https://n2t.net/ark:/13683/prDE/rdz

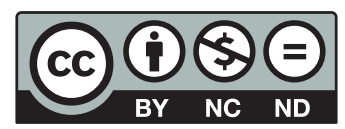




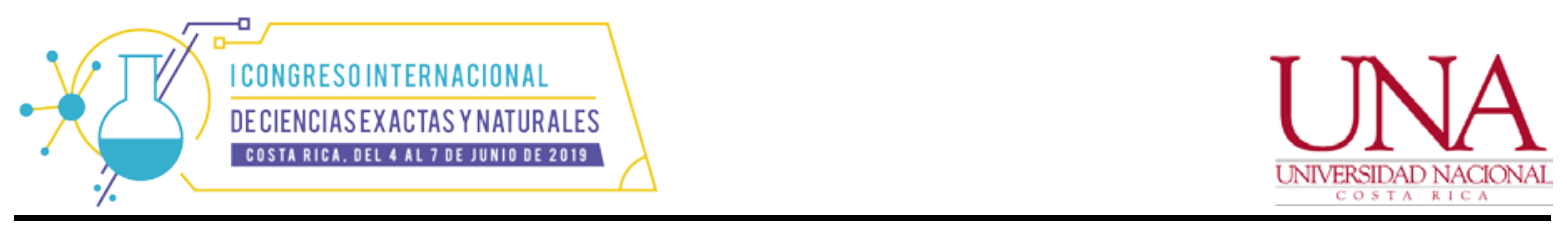

\title{
Uso de GeoGebra para la construcción de diagramas de cuerpo libre y editor de imágenes en la enseñanza de la Física
}

\author{
Marco Vinicio López-Gamboa \\ mlopez@iscr.com \\ Centro de Investigación y Gestión en Educación, Tecnología e Innovación \\ I.S Corporation \\ Neuro Aula \\ Costa Rica \\ M. Esteban Corrales-Quesada \\ escorrales@itcr.ac.cr \\ Instituto Tecnológico de Costa Rica \\ Costa Rica
}

El GeoGebra es un software diseñado principalmente para la enseñanza de la Matemática en cualquier nivel educativo, es de código abierto y es gratuito; pero que además puede ser utilizado en otras áreas del conocimiento como la Física y por supuesto para la enseñanza de la misma, en este caso particular para construcción de diagramas de cuerpo libre y como un complemento para la edición de imágenes, gracias a que con GeoGebra cuenta con elementos como vectores, circunferencias, rectas paralelas y demás objetos geométricos, que como es sabido también son indispensables en la Física, tanto para explicación de conceptos, resolución de problemas, así como para la edición de material didáctico.

Palabras clave: física; software; diagrama; vector; circunferencia.

\begin{abstract}
GeoGebra is a software designed primarily for teaching of Mathematics at any educational level, it is open source and free. Can also be used in other areas of knowledge such as Physics for the construction of free body diagrams and as a complement for image editing. GeoGebra has elements such as vectors, circumferences, parallel lines and other geometric objects, essentials in Physics for explaining concepts, solving problems, and editing teaching materials.
\end{abstract}

Keywords: physics; software; diagram; vector; circumference.

Tema: Educación científica, matemática y tecnológica

Principal área: Física

López-Gamboa, M. \& Corrales-Quesada, M. (2019). Uso de GeoGebra para la construcción de diagramas de cuerpo libre y editor de imágenes en la enseñanza de la Física. En Y. Morales-López (Ed.), Memorias del I Congreso Internacional de Ciencias Exactas y Naturales de la Universidad Nacional, Costa Rica, 2019 (e167, pp. 1-8). Heredia: Universidad Nacional. doi

http://dx.doi.org/10.15359/cicen.1.45

ISBN: 978-9968-9661-6-0. 


\section{Introducción}

Es un software libre, gratuito y de fácil acceso, que puede utilizarse desde una computadora hasta un dispositivo móvil (smartphone o tablet), además de ser multi plataforma, es decir que puede ejecutarse en diversos sistemas operativos como macOS, Windows, Android, iOS y diversas distribuciones de Linux como Ubuntu y Linux Mint.

Además de presentar algunos detalles sobre GeoGebra y los diagramas de cuerpo libre en Física, se presentará como hacer uso de este software en el contexto de la construcción de diagramas de cuerpo libre y edición de imágenes en la enseñanza de la Física, utilizando este software desde su interfaz para computadoras ya sean de escritorio o laptop.

\section{Marco teórico}

\section{GeoGebra}

Losada (2007) expone que GeoGebra ha sido desarrollado principalmente por Markus Hohenwarter desde 2001, en el departamento de Didáctica de la Matemática de la Universidad de Salzburgo.

Mientras que Abánades, Botana, Escribano \& Tabera (s.f.) lo describen como un sistema de geometría dinámica, es decir que permite realizar construcciones geométricas planas que a posteriori pueden modificarse dinámicamente y que se pueden introducir ecuaciones y coordenadas directamente, además de que tiene la potencia de manejar variables vinculadas a números ofreciendo un repertorio de comandos propios del análisis matemático, aptos para tareas como identificar puntos singulares de una función.

La figura 1 es una captura de la interfase gráfica de la versión del GeoGebra para la versión de uso en una computadora:

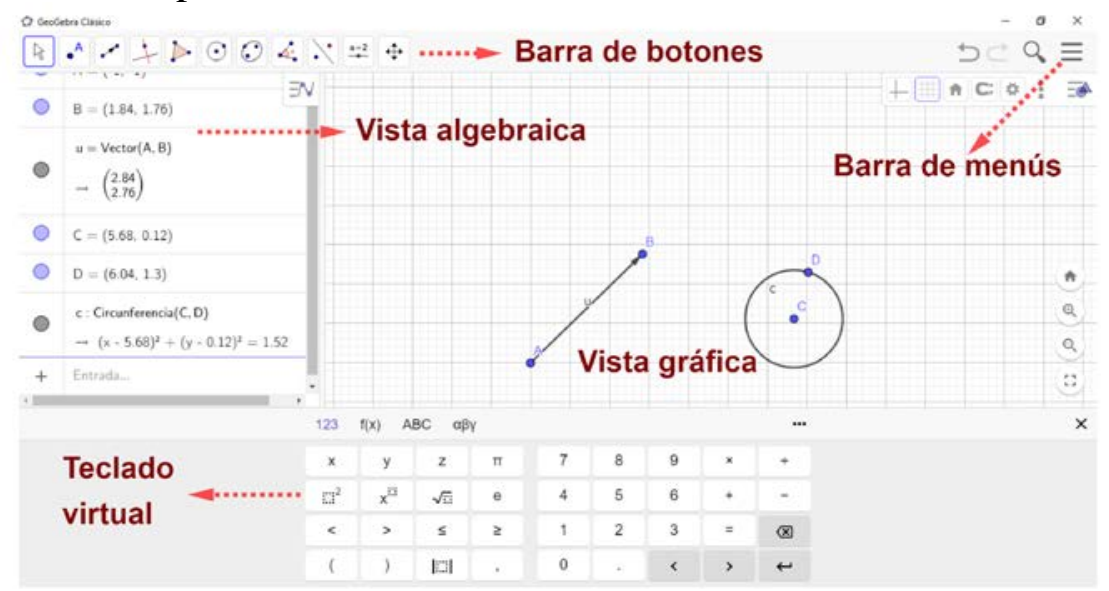

Figura 1. Interfase gráfica del GeoGebra, con algunas de sus secciones señaladas.

Fuente: los autores. 
Dentro de las características que posee este software educativo están:

- Interfaz de fácil uso (figura 1).

- Integración del código LaTeX, a la hora de insertar notación matemática.

- Posee hojas de cálculo.

- Exporta a diferentes formatos, como “.png”, “.pdf”, “.eps”, inclusive genera una dirección url de la construcción realizada.

Dentro de las versatilidades actuales, el GeoGebra permite realizar construcciones en tres dimensiones (3D):

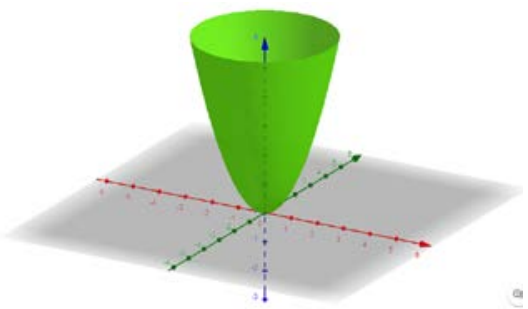

Figura 2. Muestra construcción 3D, en este caso un paraboloide.

Fuente: los autores.

Así como la implementación de la realidad aumentada (figura 3), en el caso de dispositivos móviles (smartphones y tablets), que por el momento esta disponible para los dispositivos de la marca Apple (iOS), pero que para el sistema operativo Android ya esta en proceso de desarrollo.

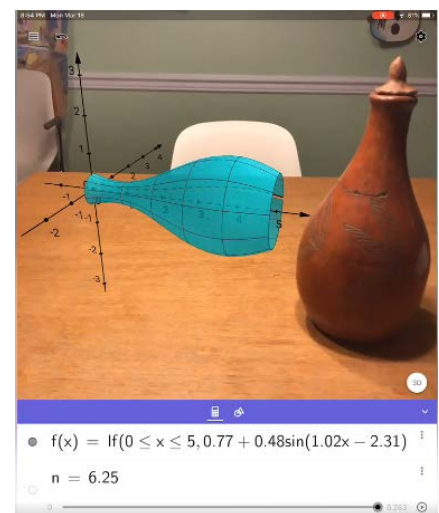

Figura 3. Realidad Aumentada por medio de GeoGebra.

Fuente: Sitio en Facebook: GeoGebra Arts \& STEAM

\section{Diagrama de cuerpo libre y demás figuras}

En la enseñanza de la Física, para representar fenómenos, explicar conceptos y en muchos ejercicios, se utilizan las gráficas, los diagramas y otras representaciones; en el caso de los diagramas de cuerpo libre (DCL), como lo definen Young y Freedman (2013), estos son una representación gráfica que muestra solamente el cuerpo elegido, "libre" de su entorno, con vectores que muestran las magnitudes y direcciones de todas las fuerzas aplicadas sobre el cuerpo por todos los cuerpos que interactúan con él. Estos ayudan a dar una mejor interpretación del concepto o conceptos y/o de la situación expuesta en un 
ejercicio en particular, como lo indica Ócariz (2000) la resolución de los problemas, tanto de estática como de dinámica, comienza con la elaboración de un DCL.
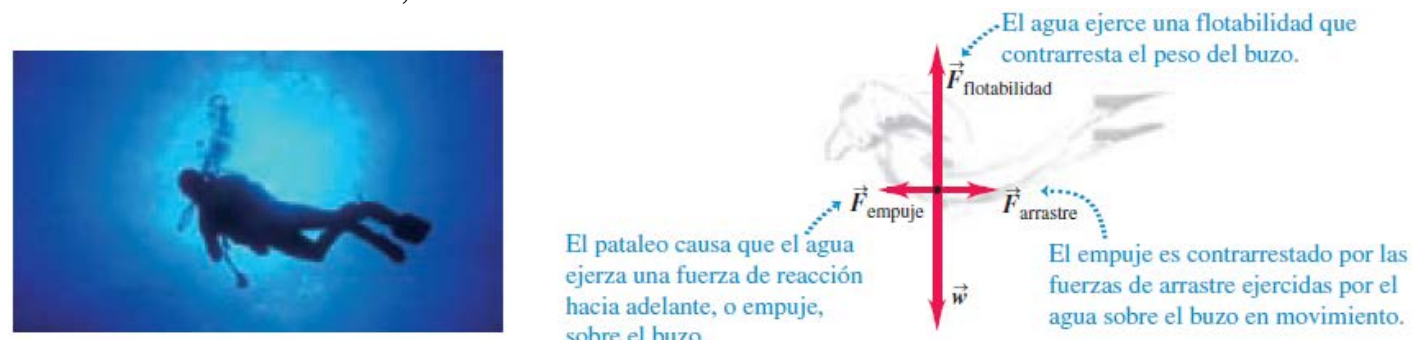

Figura 4. Muestra de imagen y su respectivo DCL.

Fuente: Young y Freedman (2013).

A nivel de la enseñanza de la Física en general se utiliza con mucha frecuencia imágenes o dibujos para representar diversas situaciones de un concepto o de un ejercicio en particular, así como sus respectivos diagramas de cuerpo libre, como lo muestra la figura 4.

\section{Metodología}

Con el GeoGebra se pueden construir imágenes o diagramas que ayuden a complementar la idea de un enunciado o la solución de un ejercicio, así como la representación gráfica de uno o varios conceptos, gracias a las diversas herramientas que ofrece como puntos, vectores, líneas rectas y diversas curvas, entre otras. A continuación, se describirá el uso del GeoGebra desde su versión para computadoras (escritorio o laptop) en la construcción y manipulación de diagramas de cuerpo libre y otras figuras en la enseñanza de la Física.

\section{Construcciones}

DCL de un plano inclinado

Se parte de un ejercicio de un examen del curso de Física General I (FS0210) de la Universidad de Costa Rica, realizado en el segundo semestre del año 2013, cuyo enunciado es:

Sobre un plano inclinado, que forma un ángulo $\alpha$ con la horizontal (figura 5), un bloque de masa $m$ es arrastrado por una cuerda hacia arriba a una velocidad constante. El coeficiente de rozamiento entre el bloque y el plano inclinado es $\mu_{\mathrm{k}}$. Determine la tensión $T$ de la cuerda, en términos de la masa m del bloque, la aceleración de gravedad $g$, el coeficiente de rozamiento $\mu_{\mathrm{k}} \mathrm{y}$ los ángulos $\alpha$ y $\beta$. 
Figura 5. Plano inclinado del enunciado.

Fuente: Examen de física general 1.

Para este caso, sin ahondar en detalles en la resolución del ejercicio se construye el diagrama de cuerpo libre:

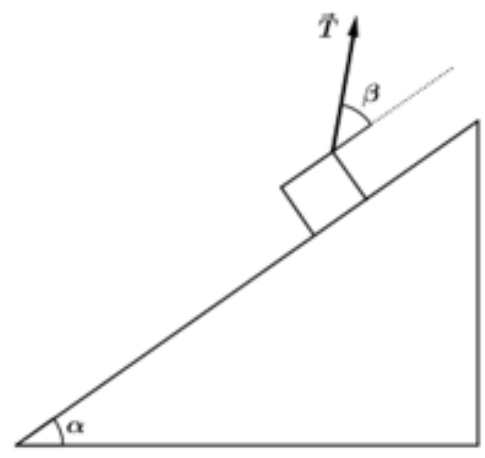

Figura 6. DCL del plano inclinado de la figura 5.

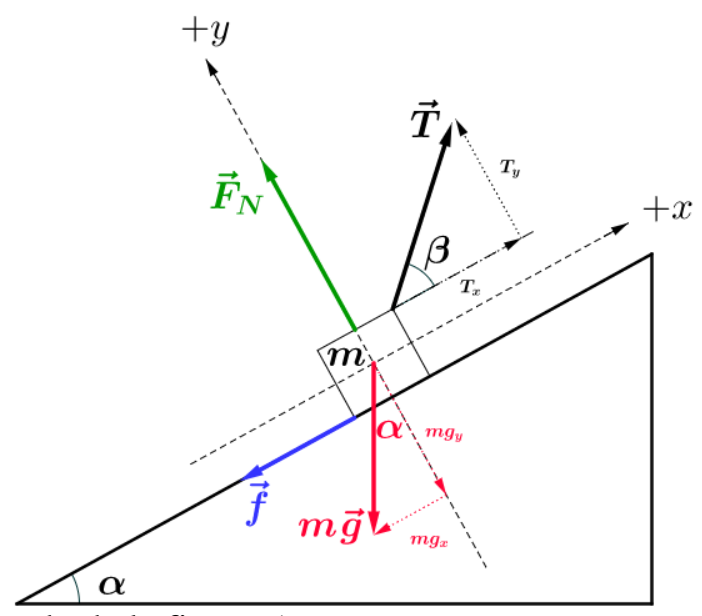

Fuente: los autores.

Básicamente para construir la figura anterior, tanto el plano inclinado como los vectores y demás elementos, se hizo uso de diferentes herramientas como las mencionadas al inicio de esta sección, así como de la intersección, ángulo dada su amplitud, segmentos, entre otras.

Para conocer y realizar una construcción similar, puede escanear el siguiente código QR y ver el video insertado en el mismo:

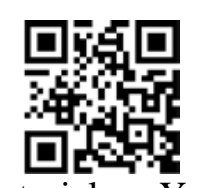

Figura 7. Código QR que remite a un video tutorial en YouTube de como hacer una construcción similar a la de la figura 6 .

Fuente: los autores. 
Se deja al lector el desarrollo de la solución de este ejercicio, cuya respuesta es:

$$
T\left(m, g, \mu_{k}, \alpha, \beta\right)=\frac{m g\left(\mu_{k} \cos \alpha+\sin \alpha\right)}{\mu_{k} \sin \beta+\cos \beta}
$$

\section{Superficies gaussianas}

Se utiliza un ejercicio de Young y Freedman (2013) sobre el tema de Ley de Gauss, el cual indica lo siguiente:

Una coraza esférica conductora, con radio interior $a$ y radio exterior $b$, tiene una carga puntual positiva $Q$ localizada en su centro. La carga total en la coraza es $-3 Q$, y está aislada de su ambiente (ver figura).

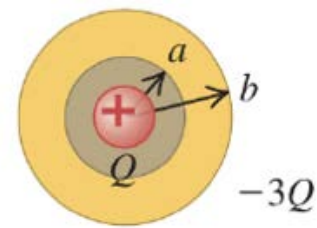

Figura 8. Imagen suministrada en el enunciado del ejercicio anterior.

Fuente: Young y Freedman (2013).

a) Obtenga expresiones para la magnitud del campo eléctrico, en términos de la distancia $r$ desde el centro, para las regiones $r<a, a<r<b$, y $r>b$.

b) ¿Cuál es la densidad superficial de carga en la superficie interior de la coraza conductora? conductora?

c) ¿Cuál es la densidad superficial de carga en la superficie exterior de la coraza

d) Elabore un diagrama de las líneas de campo y la localización de todas las cargas.

Los incisos del “a)” al “c)” se resuelven por medio del Teorema de Gauss. Mientras que el “d)”, además de usar este teorema, una representación gráfica puede ser de utilidad, utilizando de referencia la imagen dada en el enunciado, se procede por medio de las herramientas de vector y circunferencia (centro, punto), junto con el uso de rectas paralelas y perpendiculares, que son utilizadas para dar mejor exactitud como la búsqueda del centro del cascarón y que posteriormente se hacen invisibles. Para así representar a las respectivas superficies gaussianas, como se muestra en la figura 9:

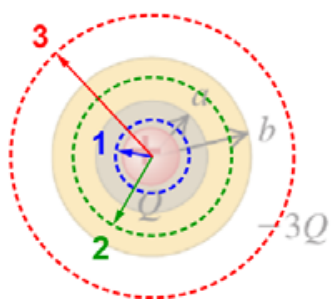

Figura 9. Imagen de coraza esférica del ejercicio anterior, con las respectivas superficies gaussianas hechas con GeoGebra.

Fuente: los autores. 
En este caso, también se hizo uso de un detalle en la configuración de la imagen, que es la opacidad, GeoGebra permite manipular el grado de opacidad no solo de los elementos que se construyan como puntos, polígonos, vectores, etc., sino además de imágenes que sean insertadas como la de figura 8 y colocadas en su vista gráfica; en particular se redujo la opacidad de la figura 6, para resaltar más a las superficies gaussianas y obtener así lo expuesto en la figura 9.

Finalmente, para representar gráficamente la respuesta del inciso “d)” por medio de GeoGebra y haciendo uso de nuevamente de vectores, circunferencia (centro, punto) y texto, entre otras; se tiene como resultado la imagen siguiente:

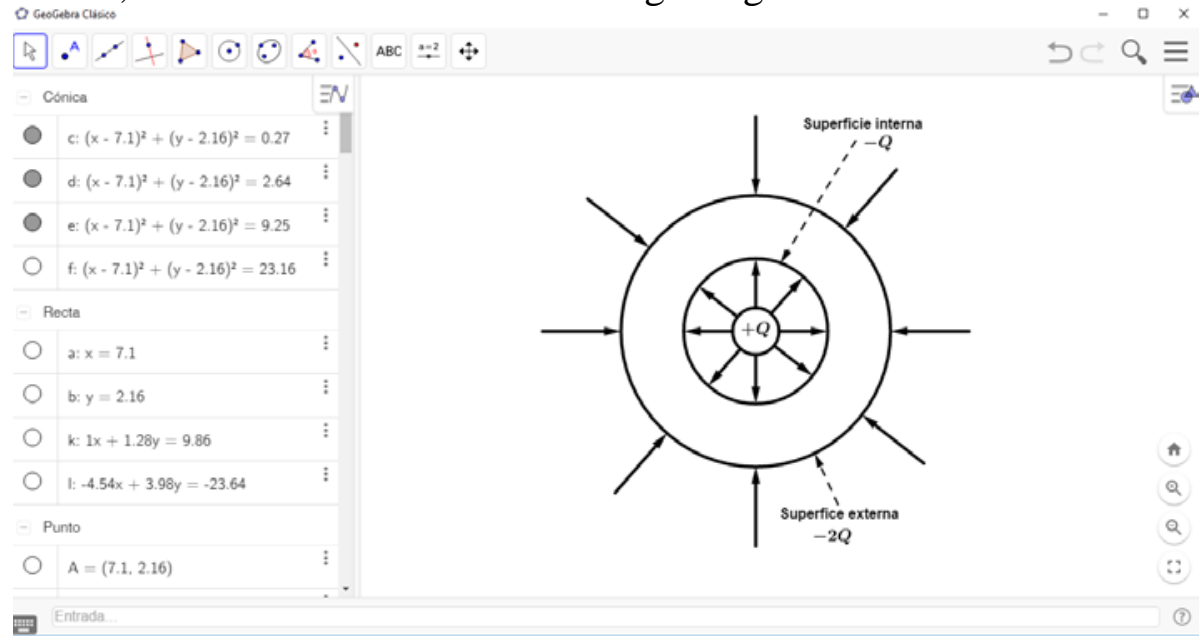

Figura 10. Diagrama de líneas de campo eléctrico, realizado con GeoGebra.

Fuente: los autores.

Se puede observar en la figura 10 parte de la interfase de GeoGebra (vista algebraica y gráfica), esto con el fin de visualizar no solo el producto terminado, en este caso el diagrama de las líneas de campo eléctrico solicitado en "d)", ubicado en la vista gráfica, sino, además, el desglose de todos elementos requeridos para su construcción desde puntos hasta ecuaciones de rectas y circunferencias, localizados en la vista algebraica, según lo representado en la figura 1.

Finalmente, para obtener las imágenes como las presentadas en las figuras 6, 9 y 10, así como cualquier otra construcción, para utilizarla en un documento de texto, presentación, etc., solo debe ubicarse en la barra de menús y hacer uso de las dos opciones que ofrece el GeoGebra, la primera es "Exportar imagen” o "Descargar como" y escoger las respectivas alternativas que ofrecen estas dos opciones, de las que se pueden resaltar que genera la imagen tanto en formato “.png” como también un archivo “.pdf”. 


\section{Análisis}

GeoGebra ofrece una gran versatilidad al momento de construir diagramas de cuerpo libre y complementar imágenes dadas con otros elementos para explicar conceptos y/o resolver ejercicios de Física, es fácil de usar, lo que permite obtener resultados como los expuestos en las figuras 6, 9 y 10; convirtiéndolo en un software útil y práctico que cualquier otro programa de construcción y edición imágenes convencional. Por esta razón, es útil para ser utilizado como una forma para generar construcciones gráficas que ilustren material didáctico como prácticas, exámenes y demás.

Es importante resaltar que, para utilizar cualquier recurso tecnológico, siempre se debe hacer un análisis contexto educativo y factibilidad, en este caso, el GeoGebra es recomendado ya que posee como se ha mencionado muchas herramientas compatibles para la enseñanza de la Física y además de ser gratuito, haciendo que sea una herramienta práctica y accesible tanto para docentes como para estudiantes.

\section{Conclusiones}

GeoGebra permite realizar diversas construcciones que sirven no solo para facilitar la labor del docente, sino, además para mejorar el proceso de enseñanza y aprendizaje de los estudiantes, este software no solo permite hacer construcciones estáticas como las expuestas en este documento, también se pueden hacer construcciones interactivas con animaciones inclusive, las cuales se puede apreciar en la siguiente sección de su sitio web: https://www.geogebra.org/materials, estas construcciones son facilitadas por diferentes docentes a nivel mundial, que las comparten para que tanto otros docentes como estudiantes las utilicen, reiterar nuevamente que GeoGebra es gratuito, lo que facilita su obtención y también como es de código abierto, esto permite que este en constante desarrollo y actualización.

\section{Referencias}

Abánades, M., Botana, F., Escribano, J., \& Tabera, L. (s.f.) Software matemático libre. La Gaceta de la RSME, 00 (0000), 0, 3-24

Recuperado de: https://bit.ly/2FmVs2u

Losada, R. (2007) GeoGebra: la eficiencia de la intuición. Real Sociedad Matemática Española.

Recuperado de: https://bit.ly/2HwldQe

Ocáriz, J. (2000) DIAGRAMAS DE CUERPO LIBRE. ¿Puede dibujarlos cualquiera? Boletín del departamento de Mecánica, 1. Universidad Nacional Autónoma de México, México.

Recuperado de: https://bit.ly/2wN0K27

Young, D., Freedman, R. (2013). Física Universitaria vol.1, 13ed. México, PEARSON.

Young, D., Freedman, R. (2013). Física Universitaria vol.2, 13ed. México, PEARSON.

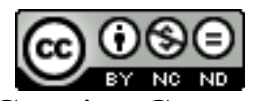

Esta obra está bajo una licencia de Creative Commons Reconocimiento-NoComercialSinObraDerivada 4.0 Internacional.

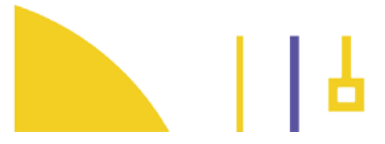

